\title{
РАВНОВЕСИЕ МЕЖДУ РАСТВОРОМ И ДВУХКОМПОНЕНТНЫМИ МОЛЕКУЛЯРНЫМИ КРИСТАЛЛАМИ
}

\author{
(Представил Ю. Канн)
}

При кристаллизации изомерных соединений, близких по структуре и химической природе, образуются, как правило, смешанные молекулярные кристаллы, в решетке которых молекулы кристаллизующихся соединений в принципе могут быть заменены друг другом. Вероятность присоединения любой молекулы к кристаллической решетке определяется разностью химических потенциалов этого соединения в жидкой фазе и поверхностном слое кристаллической решетки. Если образующиеся кристаллы являются метастабильными (скорость диффузии молекул внутри кристалла близка к нулю), то при достаточно малой скорости роста кристаллов можно допустить, что между поверхностью кристаллов и раствором в каждый момент времени имеет место равновесие. Тогда после образования одного элементарного слоя кристаллической решетки начинает строиться новый, приходящий в равновесие с раствором. Каждый последующий слой изолирует предыдущий от раствора.

При невысоких концентрациях кристаллизующихся соединений и постоянстве селективности кристаллизации примесей такой процесс описывается известным уравнением Дернера-Хоскинса.

Нами в $\left[{ }^{1-3}\right]$ изложенная выше концепция сокристаллизации примесей расширена на больший концентрационный интервал, где условия постоянства селективности обычно не выполняются. Предложен способ описания концентрационной зависимости коэффициентов распределения кристаллизующихся соединений между метастабильными кристаллами и маточным раствором с помощью простой эмпирической функции от концентрации компонентов. Этой же зависимостью в скрытой форме учитывается температурная зависимость коэффициента распределения.

В настоящей работе этот подход конкретизируется для случая образования двухкомпонентных изоморфных кристаллов. Показывается, что для этих систем из экспериментальных данных о равновесии между раствором и кристаллами можно определить функции, наиболее адекватно описывающие зависимость селективности разделения компонентов от состава раствора.

\section{Общие зависимости для поликомпонентной системы}

Рассмотрим элементарный акт кристаллизации из 1 моля растворителя, содержащего $n$ кристаллизующихся компонентов в количестве соответственно $X_{1}, X_{2}, \ldots, X_{i}, \ldots, X_{n}$ молей. Пусть между маточным раствором и смешанными метастабильными кристаллами имеет место равновесие. Обозначим коэффициенты распределения компонентов между твердой (поверхностным слоем метастабильных кристаллов) и жидкой фазами через $f_{i}\left(f_{i}=\frac{y_{i}}{X_{i}}\right.$, где $y$ - концентрация в поверхностном 
слое кристаллов, мол. доли*). Тогда балансы кристаллизующихся ко̀мпонентов при образовании одного элементарного слоя кристаллической решетки в количестве $\mathrm{d} \sum_{i=1}^{n} X_{i}$ молей задаются системой из $n$ уравнений вида

$$
\mathrm{d} X_{i}=f_{i} X_{i} \mathrm{~d} \sum_{i=1}^{n} X_{i}
$$

или

$$
\mathrm{d} \sum_{i=1}^{n} X_{i}=\frac{\mathrm{d} X_{1}}{f_{1} X_{1}}=\frac{\mathrm{d} X_{2}}{f_{2} X_{2}}=\ldots=\frac{\mathrm{d} X_{n}}{f_{n} X_{n}}
$$

Интегрирование уравнения (1) в интервалах от $\sum_{i=1}^{n} X_{i 0}$ до $\sum_{i=1}^{n} X_{i}$ и от $X_{i 0}$ до $X_{i}$ (второй индекс 0 обозначает исходный раствор) дает

$$
\sum_{i=1}^{n} X_{i 0}-\sum_{i=1}^{n} X_{i}=\int_{X_{i}}^{X_{t_{0}}} \frac{\mathrm{d} X_{i}}{f_{i} X_{i}}
$$

где $\left(\sum_{i=1}^{n} X_{i 0}-\sum_{i=1}^{n} X\right)-$ количество смешанных кристаллов.

Решение системы уравнений вида (1) или (3) при заданных (известных) $X_{10}, X_{20}, \ldots, X_{n 0}$ и $\sum_{i=1}^{n} X_{i}$ определяет концентрации всех компонентов в маточном растворе и полученных кристаллах. Но так как коэффициенты $f_{i}$ нельзя считать постоянными величинами, то решение системы уравнений (1) или (3) возможно лишь тогда, когда $f_{i}=F_{i}\left(X_{1}, X_{2}, \ldots\right.$ $\left.\ldots, X_{i} \ldots, X_{n}\right)$ известны в аналитической форме для всех компонентов.

Концентрация любого компонента в наружном слое кристаллов, находящемся в равновесни с маточным раствором, определяется активностью этого соединения в растворе. Допустим, что активность компонента задается его концентрацией $X$ и растворимостью $s$, причем активность пропорциональна концентрации в некоторой степени $r-1$ и обратной величине растворимости $\frac{1}{s}$, т. е. $a X^{r-1}$, где $a-$ коэффициент, зависящий от $\frac{1}{s}$. Учитывая, что для многокомпонентного раствора, находящегося в равновесии с наружным слоем кристаллов, должно соблюдаться условие

$$
\sum_{i=1}^{n} f_{i} X_{i}=1
$$

коэффициенты распределения $f_{i}$ можно выразить как

$$
f_{i}=a_{i} X_{i}^{r-1} / \sum_{i=1}^{n} a_{i} X_{i}^{r}
$$

где $\left(\sum_{i=1}^{n} a_{i} X_{i}^{r}\right)^{-1}-$ нормирующий множитель.

\footnotetext{
* При выражении концентрации кристаллизующихся соединений в жидкой фазе в единицах моль/моль растворителя, а в твердой фазе в мольных долях значительно упрощается математическое решение задачи. Естественно, что коэффициент распределения при таком «смешанном» способе выраження концентрации не может быть трактован как соотношение термодинамических коэффициентов активности в равновесных фазах и поэтому лишен строгого термодинамического смысла.
} 
При таком выражении $\dot{f}_{i}$ допустимо пользоваться не только абсолютными, но и относительными величинами $\frac{1}{s_{i}}$ или $a_{i}$, т. е. их взаимными соотношениями.

Нетрудно убедиться, что уравнение (5) применимо к раствору с $n=1$ (кристаллизуется только одно соединение). Действительно, тогда $X_{i}$ равен растворимости $s_{i}$ и при любой температуре $T f_{i}(T)=\frac{1}{s_{i}(T)}$. Если же $r=2$ и все $a_{i}$ пропорциональны $\frac{1}{s_{i}(T)}$, то уравнение (5) сводится к выражению (7) в [1].

Из уравнений (2) и (5) получим

$$
\frac{\mathrm{d} X_{1}}{a_{1} X_{1}^{r}}=\frac{\mathrm{d} X_{2}}{a_{2} X_{2}^{r}}=\ldots=\frac{\mathrm{d} X_{i}}{a_{i} X_{i}^{r}}=\ldots
$$

или в интегральной форме (при $r \neq 1$ )

$$
\frac{1}{a_{1}}\left(X_{1}^{1-r}-X_{10}^{1-r}\right)=\frac{1}{a_{2}}\left(X_{2}^{1-r}-X_{20}^{1-r}\right)=\ldots=\frac{1}{a_{i}}\left(X_{i}^{1-r}-X_{i 0}^{1-r}\right)=\ldots
$$

Уравнение (7) дает возможность при заданном $r(r \neq 1)$ из экспериментальных данных об изменении компонентного состава маточного раствора определить соотношение $a_{i} / a_{j}$ для любой пары компонентов $i$ и $j$. Если же $r$ также неизвестен, то решение этой задачи возможно лишь в том случае, если число кристаллизующихся соединений равно двум.

\section{Системы с двумя кристаллизующимися компонентами}

Выведем уравнения, связывающие концентрации компонентов с суммарной концентрацией кристаллизующихся соединений в равновесном маточном растворе при $n=2$.

Обозначим $\sum_{i=1}^{2} X_{i}$ через $\Sigma X$. Тогда уравнение (1) для компонента 1 выражается в виде

$$
\mathrm{d} X=\frac{a_{1} X_{1}^{r}+a_{2} X_{2}^{r}}{a_{1} X_{1}^{r}} \mathrm{~d} X_{1}
$$

Введем вспомогательную переменную $t=\Sigma X / X_{1}$ и заменим $X_{2}=$ $=\Sigma X-X_{1}$. Тогда с учетом

$$
\mathrm{d} \Sigma X=X_{1} \mathrm{~d} t+t \mathrm{~d} X_{1}
$$

получим

$$
\frac{\mathrm{d} t}{(1-t)\left[1-\frac{a_{2}}{a_{1}}(t-1)^{r-1}\right]}=\frac{\mathrm{d} X_{1}}{X_{1}}
$$

интегрирование которого в интервалах от $X_{10}$ до $X_{1}$ и от $t_{0}$ до $t$ при известных $t_{0} \equiv \Sigma X_{0} / X_{10}$ и $X_{10}$ дает зависимость между $\Sigma X$ и $X_{1}$.

Рассмотрим решение уравнения (9) при некоторых целочисленных значениях $r$. 
1. $r=0$. В этом случае

$$
X_{1}-X_{10}=\frac{a_{1}}{a_{2}}\left(X_{2}-X_{20}\right)
$$

$$
\frac{\mathrm{d} t}{1+\frac{a_{2}}{a_{1}}-t}=\frac{\mathrm{d} X_{1}}{X_{1}},
$$

интегрирование которого дает

$$
\ln \frac{1+a_{2} / a_{1}-t_{0}}{1+a_{2} / a_{1}-t}=\ln \frac{X_{1}}{X_{10}}
$$

или

$$
X_{1}=\frac{X_{10}\left(1+a_{2} / a_{1}\right)-\left(\sum X_{0}-\Sigma X\right)}{1+a_{2} / a_{1}} .
$$

Аналогично получим

$$
X_{2}=\frac{X_{20}\left(1+a_{1} / a_{2}\right)-\left(\sum X_{0}-\Sigma X\right)}{1+a_{1} / a_{2}} .
$$

2. $r=1$. В этом случае

$$
\ln \frac{X_{10}}{X_{1}}=\frac{a_{1}}{a_{2}} \ln \frac{X_{20}}{X_{2}}
$$

и

$$
\frac{\mathrm{d} t}{1-t}=\left(1-a_{2} / a_{1}\right) \frac{\mathrm{d} X_{1}}{X_{1}},
$$

интегрирование которого дает

$$
\ln \frac{1-t_{0}}{1-t}=\left(a_{2} / a_{1}-1\right) \ln \frac{X_{10}}{X_{1}}
$$

или

$$
\ln \frac{\sum X_{0}-X_{10}}{\sum X-X_{1}}=\frac{a_{2}}{a_{1}} \ln \frac{X_{10}}{X_{1}} .
$$

Аналогично получим

$$
\ln \frac{\sum X_{0}-X_{20}}{\sum X-X_{2}}=\frac{a_{1}}{a_{2}} \ln \frac{X_{20}}{X_{2}},
$$

откуда при заданной $\Sigma X$ значения $X_{1}$ и $X_{2}$ могут быть рассчитаны путем последовательного приближения.

3. $\boldsymbol{r}=2$. В этом случае

$$
\begin{gathered}
\frac{1}{X_{1}}-\frac{1}{X_{10}}=\frac{a_{1}}{a_{2}}\left(\frac{1}{X_{2}}-\frac{1}{X_{20}}\right) \\
\frac{\mathrm{d} t}{(1-t)\left[1+a_{2} / a_{1}(1-t)\right]}=\frac{\mathrm{d} X_{1}}{X_{1}},
\end{gathered}
$$

интегрирование которого дает 


$$
\ln \frac{\left(t_{0}-1\right)\left[a_{2} / a_{1}(t-1)-1\right]}{(t-1)\left[a_{2} / a_{1}\left(t_{0}-1\right)-1\right]}=\ln \frac{X_{1}}{X_{10}}
$$

или

$$
\frac{\left(\sum X_{0}-X_{10}\right)\left[\sum X-X_{1}\left(1+a_{1} / a_{2}\right)\right]}{\left(\sum X-X_{1}\right)\left[\sum X_{0}-X_{10}\left(1+a_{1} / a_{2}\right)\right]}=\frac{X_{1}}{X_{10}} .
$$

Аналогично получим

$$
\frac{\left(\sum X_{0}-X_{20}\right)\left[\sum X-X_{2}\left(1+a_{2} / a_{1}\right)\right]}{\left(\sum X-X_{2}\right)\left[\sum X_{0}-X_{20}\left(1+a_{2} / a_{1}\right)\right]}=\frac{X_{2}}{X_{20}} .
$$

Если $r>2(r \in N)$, то уравнение (9) можно также интегрировать известными методами, снижая в каждой ступени интегрирования степень полинома под интегралом на единицу. После $(r-2)$-го интегрирования подынтегральная часть тождественна левой половине уравнения (21).

4. $\boldsymbol{r} \in \boldsymbol{R}(\boldsymbol{r} \notin \boldsymbol{N})$. В этом случае уравнение (9) не интегрируется. В системе с двумя кристаллизующимися компонентами зависимость между $\Sigma X$ и концентрацией отдельных компонентов может быть определена путем последовательного приближения. Исходим из уравнения (7) в виде

$$
X_{1}^{1-r}-X_{10}^{1-r}=\frac{a_{1}}{a_{2}}\left(X_{2}^{1-r}-X_{20}^{1-r}\right),
$$

откуда при заданном нецелочисленном $r$ по набору опытных величин $X_{1}$ и $X_{2}$ определяем $a_{1} / a_{2}$, с помощью которого для любой $\Sigma X$ легко найти $X_{1}$ и $X_{2}$, удовлетворяющие уравнению (25) и требованию $X_{1}+X_{2}=\Sigma X$.

Если же $r$ также неизвестен, то путем последовательного приближения определяем такое его значение, при использовании которого совместно с соответствующим ему $a_{1} / a_{2}$, полученным по уравнению $(25)$, достигается наилучшее согласие экспериментальных и расчетных величин $X_{1}$ и $X_{2}$. Такое приближение трудоемко, но в принципе также легко осуществимо.

Таким образом, полученные зависимости дают возможность на основе экспериментальных данных о кристаллизации смеси двух соединений опредслить параметр $r$ и соотношение $a_{1} / a_{2}$. С помощью этих величин можно прогнозировать составы равновесных кристаллов и маточного раствора при любом выходе кристаллов и любом составе исходной кристаллизующейся смеси.

Автсры благодарны Х. Тамвелиусу за ценные замечания.

\section{ЛИТЕРА А У РА}

1. Мельдер Л. И., Забеллевич И. В., Тамвелиус Х. Я. Равновесие между поликомпо. нентным раствором и метастабильными молекулярными кристаллами // Ж. прикл. хим., 1988, 61, № 4, 762-766.

2. Мельдер Л. И., Забеллевич И. В. Кристаллизащия алкилрезорцинов и их олигомеров из раствора // Тезисы респ. науч. конф. «Химия и примененне фенолальдегидных смол». Таллин, $1987,23-24$.

3. Забеллевич Н. В., Мельдер Л. Н. Кристаллизация алкилрезорцинов из раствора // Тезисы докл. Всесоюз, науч.-техн. совещ. «Совершенствование технологин сланцепереработки и новые направления использования сланцепродуктов». КохтлаЯрве, 1988, 81.

Ннститут химии

Академии наук Эстонской ССР
Поступила в редакцию 14/XI 1988 


\section{LAHUSE JA KAHEKOMPONENDILISTE MOLEKULAARKRISTALLIDÉ} VAHELINE TASAKAAL

On saadud võrrandid, mis kirjeldavad kahekomponendiliste molekulaarkristallide ja emalahuse koostise vahelist seost, kui kristallid on isomorfsed ja metastabiilsed. Seejuures eeldatakse, et molekuli kristallvõrele liitumise tõenäosus on võrdeline tema keemiliste potentsiaalide vahega vedelfaasis ja kristallide pindkihis. On näidatud, et kristalliseeruvate ühendite jaotuskoefitsiente kirjeldava võrrandi parameetrid on määratavad katseandmete põhjal.

\section{MOLDER, I. ZABELLEVICH}

\section{EQUILIBRIUM BETWEEN THE SOLUTION AND BINARY MOLECULAR CRYSTALS}

For the binary systems containing isomorphic and metastable molecular crystals in mother solution, the equations for the compositions of solid and liquid phases have been derived. It has been assumed that the probability of the addition of molecules to crystal lattice is proportional to the difference of chemical potentials in liquid phase and surface layer of crystals. In this case the coefficients of distribution (f) of the crystallizing compounds can be described by Eq. (5) in which $X$ is concentration in the mother solution (molality) and $a$ and $r$ are empiric constants. The dependence between the concentrations of components $X_{1}$ and $X_{2}$ and their summary concentration $\Sigma X$ has been described using Eqs (13) and (14), (18) and (19), (23) and (24) for the cases $r=0, r=1$ and $r=2$ respectively. If $r$ is fractional number, the iteration by Eq. (25) has to be applied.

The given equations can be used to find the values of the parameter $r$ and the relation $a_{1} / a_{2}$ on the basis of experimental data of the crystallization. By means of these values the composition of crystals and mother solution in equilibrium conditions can be predicted for any yield of the crystals and any composition of the initial solution. 